\title{
THE NEED FOR NEW NEUTRINO PHYSICS OR A COOLER SUN IN THE SOLAR NEUTRINO PROBLEM
}

\author{
X. Shi and D.N. Schramm
}

\section{Abstract}

It is shown that the current solar neutrino situation, now that we have the SAGE and GALLEX result along with the results from the Kamiokande and the Homestake experiments, is unfortunately still quite ambiguous. The differences between observations and the standard solar theory may still be due to either astrophysical inputs or new neutrino physics. In particular, the astrophysical solution, which requires a cooler Sun than the standard solar model of Bahcall et al., may still be viable. The need for new neutrino physics, MSW or vacuum neutrino mixing, is sensitive to the results of the Homestake experiment and SAGE. The use of future experiments, SNO, Borexino, the Super Kamiokande, and the Iodine experiment to resolve this ambiguity are explicitly discussed.

\section{Introduction}

The deficit of the solar neutrino flux with respect to the prediction of the standard solar models, observed by the current solar neutrino experiments, is one of the major issues of modern physics [1,2]. Currently, the Homestake experiment [3], the Kamiokande II and III experiments [4], the SAGE experiment [5], and the GALLEX experiment [6], all observed neutrino fluxes lower than the predictions of the standard solar model of Bahcall and Ulrich (hereafter BUSSM) [1] or the standard solar model of Turck-Chièze et al. (hereafter TSSM) [7]. The current solar neutrino experimental status and the theoretical predictions are shown in Table 1. The spectrum of solar neutrinos from different reactions calculated by BUSSM is shown in fig. 1 [1].

There are two basic approaches used to explain the deficit. One is new neutrino physics, and the other is to modify the solar model. In the first approach, the MSW matter mixing is the most natural and robust scenario of new physics [8], although vacuum neutrino oscillations are also allowed but with a narrower range in the parameter space [9]. Under the MSW matter mixing scheme, solar neutrinos $\left(v_{e}\right)$ mix with other neutrino species $\left(v_{\mu}\right.$ or $\nabla_{\tau}$, or new hypothetical sterile neutrinos). On their way out of the sun, $v_{e}$ 's encounter a resonance inside the sun and flip their flavor. To reproduce the fluxes observed by the current solar neutrino experiments, the neutrino mixing parameters are restricted to the diagonal and vertical regions of a triangle in the parameter space $\left(\Delta m^{2}=m_{2}^{2}-m_{1}^{2}\right.$ vs. $\sin ^{2} 2 \theta_{e x} / \cos 2 \theta_{e x}$, where $m_{1}$ and $m_{2}$ are the neutrino mass eigenvalues and $\theta_{e x}$ is the mixing angle between $v_{e}$ and $v_{x}$ ), assuming standard solar models $[10,11]$. There are also other proposed new physics solutions, such as the OVV neutrino magnetic moment solution [12] and its extension, spin-flavor precession $[13,14]$, and the neutrino decay solution $[15]$, etc.
For the second approach, the simplest technique is to modify the astrophysical input parameters sufficiently to fit the experimental results [2]. The current standard solar models have large uncertainties in certain input parameters. Different choices of input parameters could yield different neutrino fluxes, especially for ${ }^{8} \mathbf{B}$ neutrinos. This is manifested in the comparison of the predictions of the BUSSM and the TSSM (shown in Table 2). The differences in the neutrino fluxes in these two models are mainly caused by the different rates adopted for the reaction ${ }^{7} \mathrm{Be}(p, \gamma)^{8} \mathrm{~B}$ and the reaction ${ }^{3} \mathrm{He}\left({ }^{3} \mathrm{He}, 2 \mathrm{p}\right){ }^{4} \mathrm{He}[1,7,16]$. When the same input values are used, the two models (and in fact, the standard solar models of Sienkiewicz et al. [17], Filippone and Schramm [18], and of the Princeton-Yale collaboration [16]) all agree with each other within $0.1 \mathrm{SNU}$ for the ${ }^{37} \mathrm{Cl}$ experiment [16]. Similar agreements are also found for other experiments. Therefore, simply because of the uncertainties in the input parameters instead of the underlying physics of the solar models or the calculation methods exploited, different predictions for the solar neutrino experiments can be drawn. However such input parameter variations cannot cause the less temperature sensitive ${ }^{7} \mathrm{Be}$ and especially $p p$ neutrino fluxes to be significantly decreased relative to the ${ }^{8} \mathrm{~B}$ neutrino flux. Thus, for example, if it is eventually shown that the ${ }^{7} \mathrm{Be}$ neutrino flux is depleted more than the ${ }^{8} \mathrm{~B}$ neutrino flux, then new neutrino physics is required. At present, the comparison of the ${ }^{37} \mathrm{Cl}$ experiment (sees ${ }^{8} \mathrm{~B}$ neutrinos and ${ }^{7} \mathrm{Be}$ neutrinos) to the Kamiokande experiments (see ${ }^{8} \mathrm{~B}$ neutrinos only) and GALLEX as well as SAGE (see $p p,{ }^{7} \mathrm{Be}$ and ${ }^{8} \mathrm{~B}$ neutrinos) might, at face value, imply a greater ${ }^{7} \mathrm{Be}$ neutrino reduction, and thus new neutrino physics. But one should be cautious here as we shall see.

Besides the theoretical uncertainties in the solar models, questions have occasionally been cast on the solar neutrino experiments themselves [19]. Because of the weakness of 
Table 1

Current solar neutrino experimental status

\begin{tabular}{|c|c|c|c|}
\hline & Homestake & Kamiokande & Gallium exp. \\
\hline Detector material & $\mathrm{C}_{2} \mathrm{Cl}_{4}$ & $\mathrm{H}_{2} \mathrm{O}$ & $\begin{array}{c}\mathrm{GaCl}_{3}-\mathrm{HCl} \text { (GALLEX) } \\
\mathrm{Ga} \text { (SAGE) }\end{array}$ \\
\hline Fiducial mass & 610 ton & 680 ton & $\begin{array}{c}30 \text { ton Ga (GALLEX) } \\
57 \text { ton (30t before 1991) (SAGE) }\end{array}$ \\
\hline Reaction & $\begin{array}{c}{ }^{37} \mathrm{Cl}\left(v_{e}, e^{-}\right)^{37} \mathrm{Ar} \\
v_{e} \text { only }\end{array}$ & $\begin{array}{l}\text { v-e scattering } \\
v_{e}, v_{\mu}, v_{5}, \bar{v}\end{array}$ & $\begin{array}{c}{ }^{71} \mathrm{Ga}\left(v_{e}, e^{-}\right){ }^{71} \mathrm{Ge} \\
v_{e} \text { only }\end{array}$ \\
\hline Threshold & $0.814 \mathrm{MeV}^{a}$ & $7.5 \mathrm{MeV}^{h}$ & $0.233 \mathrm{MeV}^{a}$ \\
\hline Type & $\begin{array}{l}\text { Radiochemical } \\
\text { Time Integral } \\
\text { Energy Integral }\end{array}$ & $\begin{array}{l}\text { Direct Counting } \\
\text { Real Time } \\
\text { Spectroscopic }\end{array}$ & $\begin{array}{l}\text { Radiochemical } \\
\text { Time Integral } \\
\text { Energy Integral }\end{array}$ \\
\hline BUSSM prediction (theoretical range) & $7.9 \pm 2.6 \mathrm{SNU}^{*}$ & $1.0 \pm 0.37^{\dagger}$ & $132_{-17}^{+20} \mathrm{SNU}$ \\
\hline TSSM prediction ( $1 \sigma$ error) & $6.4 \pm 1.3 \mathrm{SNU}$ & $0.75 \pm 0.20^{+}$ & $123 \pm 7 \mathrm{SNU}$ \\
\hline Event rate & $\sim 0.5 /$ day & $\sim 0.3 /$ day & $\sim 0.3 /$ day \\
\hline Observed flux & $2.28 \pm 0.23 \mathrm{SNU}$ & $\begin{array}{l}0.46 \pm 0.05 \pm 0.06(\mathrm{~K} \mathrm{II})^{\dagger} \\
0.55 \pm 0.07 \pm 0.06(\mathrm{~K} \text {. III })^{\dagger}\end{array}$ & $\begin{array}{r}83 \pm 19 \pm 8 \text { SNU (GALLEX) } \\
58_{-24}^{+17} \pm 14 \text { SNU (SAGE) }\end{array}$ \\
\hline
\end{tabular}

- : The threshold energy of the Incoming neutrinos.

b The threshold entergy of the recoil electrons.

$\uparrow$ Normalized by the prediction of the BUSSM.

- ISU $=10^{-36}$ event/target atom/second.

the interaction between neutrinos and matters, it is very difficulf to méasure the incoming solar neutrino fluxes: Current experiments utilize two general methods to: measure neutrino flux [2]: the radiochemical method and the neutrino-electron scattering method: So far, even with detector fiducial masses of $30-600$ tons, the average counting rates of the current radiochemical solar neutrino experiments are of the order $0.1 /$ day. To extract the real events from such small signals and such large volumes of material is an extremely challenging task. Careful calibrations of the experiments are essential. However, among the five current solar neutrino experiments, only the Kamiokande ' $v-e$ scattering experiments have been truly calibrated [20]. Therefore, the low rates with respect to the standard solar models of the current radiochemical experiments have to be taken as preliminary. However, such a statement should in no way defract from the imipressive experimèntal techniques used in each of 'the experiments and the open and informed manner in which the experiments have attempted to check each potential source of error. In this latter regard, the 20-year pioneering experiment of Davis is particularly notable.

Given all these factors that could cause ambiguity in the solar neutrino problem, the purpose of this review is to attempt to explore the following question: How necessary is new neutrino physics? In other word, under what circumstance will we have to resort to new physics to explain the solar neutrino deficit? In section 2 we will discuss some occasionally raised criticisms of the current solar neutrino experiments. In section 3, we will review the major uncertainties of the current solar models. We will discuss how by modifying the input parameters of the standard solar model, there could be minimal conflict between many of the experiments and the theory. In section 4, we will do statistical tests to show how well the MSW matter mixing solution (representing the new neutrino physics) and modified solar models each fit the experimental results. In section 5 , we will discuss future solar neutrino experiments, and show their roles of determining the need for the new neutrino physics and resolving the ambiguity. 


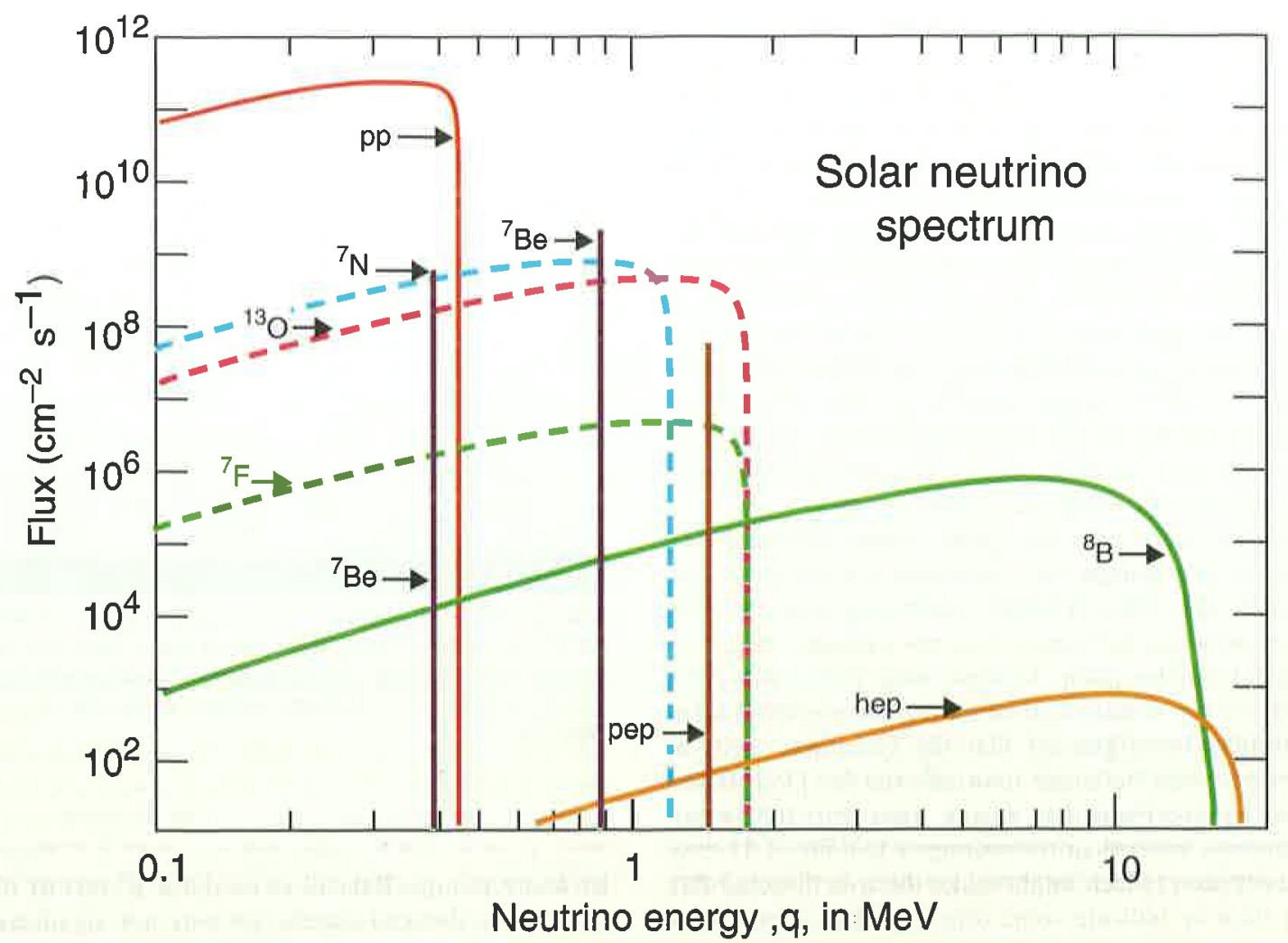

\section{FICIURE}

The solar neutrino spectrum that is predicled by BUSSM. The flux is given in $\mathrm{cm}^{-3} \mathrm{~s}^{-1}$ at 1 astronomical unit (Courtesy. of J.N. Bahcall). The flux of hep neutrinos should be reduced by a factor of 6.15 based on the 1992 model of Bahcall et al. [16].

Table 2

Solar neutrino fluxes predicted by BUSSM and TSSM.

\begin{tabular}{|c|c|c|c|}
\hline \multirow[b]{2}{*}{$\begin{array}{l}\text { Neutrino } \\
\text { Sources }\end{array}$} & \multicolumn{3}{|c|}{ BUSSM $\left[\mathrm{TSSM}^{\dagger}\right]$ Prediction } \\
\hline & $\begin{array}{l}{ }^{37} \mathrm{Cl} \text { exp. } \\
\text { (SNU) }\end{array}$ & $\begin{array}{l}{ }^{71} \text { Ga exp. } \\
\text { (SNU) }\end{array}$ & $\begin{array}{l}\text { Kamiokande } \\
(\times \text { BUSSM) }\end{array}$ \\
\hline$p p$ & $\begin{array}{ll}0 & {[0]}\end{array}$ & $70.8[71.1]$ & $0 \quad[0]$ \\
\hline pep & $0.2[0.2]$ & $3.0[2.8]$ & $\begin{array}{ll}0 & {[0]}\end{array}$ \\
\hline${ }^{7} \mathrm{Be}$ & $1.1[1.0]$ & $34.4[30.6]$ & $\begin{array}{ll}0 & {[0]}\end{array}$ \\
\hline${ }^{8} \mathrm{~B}$ & $6.1[4.6]$ & $14.0[9.3]$ & $1.0[0.75]$ \\
\hline${ }^{13} \mathrm{~N}$ & $0.1[0.1]$ & $3.8[3.9]$ & 0 \\
\hline${ }^{15} \mathrm{O}$ & $0.3[0.4]$ & $6.0[6.5]$ & $0 \quad[0]$ \\
\hline Total & $7.9[6.4]$ & $132 \quad[123]$ & $1.0[0.75]$ \\
\hline
\end{tabular}

† Based on 1992 model of Turck-Chièze et al. [7].

\section{The Solar Neutrino Experiments}

There are five running solar neutrino experiments: the Kamiokande neutrino-electron scattering experiments (including the Kamiokande II and the Kamiokande III), the Homestake chlorine capture experiment, the SAGE gallium capture experiment and the GALLEX gallium capture experiment [21]. As mentioned above, only the Kamiokande experiments has been fully calibrated. In order to calibrate the gallium experiments, an $\mathrm{MeV} / \beta$-decay source $\left({ }^{51} \mathrm{Cr}\right)$ with a strength of $\sim 1$ megacurie is needed. GALLEX plans such a calibration beginning in October, 1993, and SAGE is also planning a calibration in the not-too-distant future. No sufficiently energetic and luminous $\beta$-source exists for calibrating the ${ }^{37} \mathrm{Cl}$ experiment. But calibration has been proposed using LAMPF to expose a similar chlorine tank with energetic neutrinos.

The chlorine capture experiment at Homestake uses 610 tons of $\mathrm{C}_{2} \mathrm{Cl}_{4}$ to absorb incoming neutrinos by: 
The ${ }^{37} \mathrm{Ar}$ subsequently decays via $\mathrm{K}$-shell electron capture with a half life of 35-day and emits a $2.82 \mathrm{keV}$ Auger electron. Therefore, clear signs of the ${ }^{37} \mathrm{Ar}$ production are electrons with energies $2.82 \mathrm{keV}$, fast rising electronic pulses associated with the electrons (because of their low energies and hence short ionization range with respect to $\beta$ rays, cosmic rays and compton electrons), and a decay spectrum of 35 days half-life.

The average ${ }^{37} \mathrm{Ar}$ production rate for the Homestake experiment from 1970-1990 (Run 18-109) is $0.49 \pm 0.03$ atom/day, with a background of $0.08 \pm 0.03$ atom/day [3] This corresponds to a solar neutrino flux of $2.2 \pm 0.3 \mathrm{SNU}$ (1 SNU $=10^{-36}$ capture/target atom/sec). It shows the largest reduction with respect to the standard solar models among the current solar neutrino experiments. Occasionally questions are raised regarding the Homestake experiment.

For example, there were two pump failures during 1984 85. After these new pumps were installed, the observed rate was unusually high, $3.6 \pm 0.7 \mathrm{SNU}$ averaging over 1986-88 (Run 90-99), which is $2 \sigma$ higher than the average rate $2.1 \pm$ 0.3 SNU prior to the pump failures from 1970-1984 [19]. While it may be just a statistical variation, the possible inconsistency prompts the argument that the systematic error of the experiment might be larger than allowed for [19]. It has been argued by Morrison that during Runs 101-105, which showed countings lower than the average, a half-life of 35 days was not clearly seen (which might make the true detected flux even lower then or indicate some other systematic problem), while during the previous high-counting period, Runs $92-100$, the 35-day half-life was obvious [19].

Various tests have been done on the Homestake experiment and no source of unanticipated systematic errors has ever been found [19]. Pump repairs prior to 1984 also indicated no sign of affecting the counting rates. Up to Run 109 , the unweighted average rate is $2.0 \pm 0.3$ SNU before the pump failures (compared with $2.1 \pm 0.3 \mathrm{SNU}$ obtained from the maximal likelihood method [22]), and $2.8 \pm 0.6 \mathrm{SNU}$ after the failures (the maximal likelihood calculation is not yet published for this data subset) [23]. Simply from the unweighted averages, the purported discrepancy before and after the pump failures has now decreased to a statistically insignificant level. Therefore the unusual high capture rate right after the pump failures is probably just due to statistical fluctuations, but shifts in sets of runs are curious.

This brings to mind another debated issue about the Homestake experiment which is that some claimed it shows an anti-correlation between the number of sunspots and the counting rates [24], which was not seen by the Kamiokande II during the period $1987-90$, when there was a major change in number of sunspots. Fig. 2 shows the data of the two experiments and the average sunspot numbers during 1987-90. No significant variation is found in the data of the Kamiokande II. The existence of the anti-correlation has been investigated

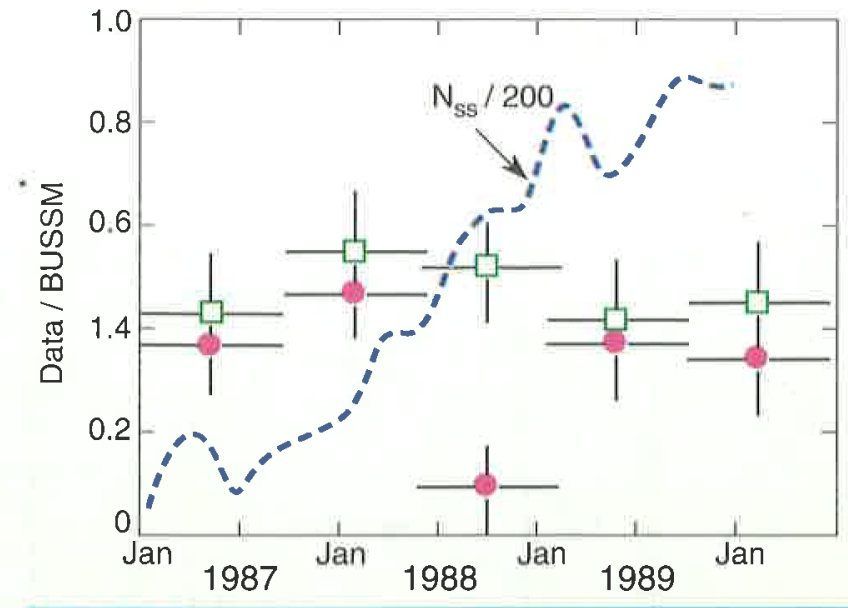

IIGLR:

Time variation of the ratio of the observed neutrino rate (from ref. 25) to the BUSSM prediction for the Kamiokande II (open square) and the chlorine experiment (solid diamond), and the monthly averaged sunspot numbers $N_{\mathrm{ss}}$ (the dashed line, data from Solar Geophysical Data prompt reports), during the period 1987-1990.

by many groups. Bahcall et al. did a $\chi^{2}$ test to the data and found that the anti-correlation was not significant, and was very sensitive to the errors of the data as well as the confidence of the very low counting runs [25]. Filippone and Vogel used a maximum likelihood technique and found the same conclusion [26]. Therefore the anti-correlation cannot be either confirmed or ruled out conclusively. This remains true even after the uncertainty of sunspot numbers is included if one thinks the solar neutrino flux actually directly correlates with the solar magnetic field [27]. Shi, Schramm, Roser and Dearborn [14] have shown that, given the current conservative limits on the neutrino magnetic moment and the solar magnetic field in the convective zone, the neutrino magnetic moment solution and the resonant spin-flavor precession solution to the solar neutrino problem fail to yield variations over the solar cycle that are large enough to be observable in the chlorine experiment. Therefore any significant anticorrelation with the sunspots (or for that matter any variation from a constant flux) in the chlorine experiment may alternatively suggest unknown systematic effects in the experiment itself. Also, if the anti-correlation does exist, Morrison has argued that its absence in the Kamiokande II experiment might be indicative of intermittent experimental problems in the chlorine experiment [19]. While the significance of a correlated time variation is debatable, most statistical analyses done to date do indicate a non-negligible but unfortunately low probability that the data is from a constant counting rate 
[25-28]. The possible time variation shown in the data of the Homestake experiment with respect to the more or less constant huxes observed by the Kamiokande II might suggest some unexpected systematic errors in the Homestake experiment. However, as mentioned above, no one has been able to indentify the sources of such a variation despite repeated attempts and variety of tests.

The two gallium capture experiments, SAGE and GALLEX, use the reaction

$$
\nu_{e}+{ }^{71} \mathrm{Ga} \rightarrow e^{-}+{ }^{71} \mathrm{Ge}, E_{\mathrm{lh}}=0.233 \mathrm{MeV},
$$

to capture neutrinos. ${ }^{71} \mathrm{Ge}$ subsequently decays with a half-life of 11 days. The gallium experiments have the advantage that they can observe the most abundant and the least uncertain solar $p p$ neutrinos. Beside the fact that neither of them have been fully calibrated, some have occassionally raised questions about the SAGE experiment [19]. Three of their first five runs have best fits of $0 \mathrm{SNU}$, and none of these five runs has observed the 11-day half-life in the ${ }^{71} \mathrm{Ge}$ decay spectrum [5]. Also the statistics are too low to draw significant conclusions. Even so, the early SAGE result $\left(20_{-20}^{+15} \pm 32 \mathrm{SNU}\right)$ is still in statistical agreement with the GALLEX result $(83 \pm 19 \pm 8$ SNU) at the $2 \sigma$ level. The recent 6 runs of SAGE yielded an average rate of $85_{-32}^{+22} \pm 20 \mathrm{SNU}$, which agrees extremely well with the GALLEX result [5]. The overall average of SAGE is $58_{-24}^{+17} \pm$ 14 SNU [5]. The SAGE collaboration has also questioned the GALLEX estimate of systematic errors, suggesting that the \pm 8 SNU might be increased significantly, perhaps as much as a factor of $2[5]$.

\section{Solar Models}

The algorithm of solar models is to evolve a 1 solar mass protostar (a homogeneous cloud of hydrogen and hellium with a small admixture of heavier elements) from about 4.6 billion years ago to now to match the current solar luminosity and radius $[1,2]$. The structure of the Sun is divided into two physical zones: the inner radiative zone, where the energy gen- erated at the solar core is transferred by radiation; and an outer convective zone, where the energy is transferred by turbulant convection. The convective zone is described by a single parameter, the mixing length, which is roughly the size of the density scale height. The mixing length, together with the helium abundance, are two free input parameters to adjust in the standard solar models to match the current Sun. Other major input parameters are, the heavy element abundance, the radiative opacity, the nuclear reaction parameters, the solar age, and the equation of state. The details are thoroughly reviewed in Bahcall's book "Neutrino Astrophysics" and sensitivity to assumptions has been systematically explored by Bahcall and his collaborators in a series of papers over the last three decades.

Table 3 lists the major different input parameters and results of the BUSSM, TSSM, the improved model of Bahcall et al. (with helium diffusion) and a modified solar model which will be explained at the end of the section. The improved model of Bahcall et al. (1992) is based on the BUSSM but with improved input parameters [16]. Without considering helium diffusion, it yields $7.2 \pm 2.7$ SNU for the chlorine experiment and $127_{-16}^{+19}$ SNU for gallium experiments. After taking into account helium diffusion which is not treated in any of the other solar models, the model yields $8.0 \pm 3.0 \mathrm{SNU}$ and $132_{-17}^{+21}$ SNU respectively. $S_{0}$ in table 3 represents the cross section of each nuclear reaction. It is related to the cross section $\sigma(E)$ by [2]

$$
S(E)=\sigma(E) E \exp (2 \pi \eta) \text { and } S_{0}=S(0) .
$$

The quantity $\eta=Z_{1} Z_{2}\left(e^{2} / \hbar v\right)$, where $Z_{1}$ and $Z_{2}$ are the charges of colliding particles, and $v$ is their relative speed.

The standard solar models have been very successful in finding agreement with the astronomical observations, for example, the spectrum for the $p$-mode oscillation of the Sun [1]. (The only exception is the prediction of the amount of the solar surface ${ }^{7} \mathrm{Li}$ depletion relative to observations; this discrepancy is presumably due to the treatment of convective overshoot at the convective/radiative boundary and should

Table 3

Major different inputs and the resultant $T_{c}$ and ${ }^{37} \mathrm{Cl}$ rate for different standard solar models.

\begin{tabular}{|l|ccccc|}
\hline Models & $S_{0}\left({ }^{7} \mathrm{Be}+p\right)$ & $S_{0}\left({ }^{3} \mathrm{He}+{ }^{3} \mathrm{He}\right)$ & $Z$ & $T_{c}$ & ${ }^{37} \mathrm{Cl}$ rate \\
\hline BUSSM & $24.3 \mathrm{eV} \cdot \mathrm{b}$ & $5.15 \mathrm{MeV} \cdot \mathrm{b}$ & 0.0196 & $1.564 \times 10^{9} \mathrm{~K}$ & $7.9 \mathrm{SNU}$ \\
TSSM & $21 \mathrm{eV} \cdot \mathrm{b}$ & $5.57 \mathrm{MeV} \cdot \mathrm{b}$ & 0.0197 & $1.551 \times 10^{9} \mathrm{~K}$ & $6.4 \mathrm{SNU}$ \\
Bahcall et al. 1992 (with helium diffusion) & $22.4 \mathrm{eV} \cdot \mathrm{b}$ & $5.57 \mathrm{MeV} \cdot \mathrm{b}$ & 0.0196 & $1.567 \times 10^{9} \mathrm{~K}$ & $8.0 \mathrm{SNU}$ \\
Present modified model & $20.2 \mathrm{eV} \cdot \mathrm{b}$ & $5.57 \mathrm{MeV} \cdot \mathrm{b}$ & 0.0150 & $1.545 \times 10^{9} \mathrm{~K}$ & $4.5 \mathrm{SNU}$ \\
\hline
\end{tabular}


have no effect on solar neutrinos [29].) But it has relatively large uncertainties in predicting the solar neutrino fluxes, especially the ${ }^{8} \mathrm{~B}$ neutrino flux and $\mathrm{CNO}$ neutrino fluxes, due to their sensitivity to the uncertainties of the input parameters. Table 4 shows the uncertainties in the solar neutrino flux prediction of BUSSM, TSSM and Bahcall's improved model with helium diffusion $[1,7,16]$. Among the major neutrino sources, the ${ }^{8} \mathrm{~B}$ neutrino flux clearly has the largest uncertainty. Since three of the current solar neutrino experiments, the chlorine experiment and the two Kamiokande experiments, are mostly or solely sensitive to the ${ }^{8} \mathrm{~B}$ neutrino flux, their theoretical implication is greatly obscured by the uncertainties in the neutrino flux prediction.

\section{Table 4}

Solar neutrino fluxes and their uncertainties predicted by different standard solar models

\begin{tabular}{|c|c|c|c|}
\hline $\begin{array}{c}\text { Neutrino } \\
\text { Flux } \\
\left(10^{8} \mathrm{~cm}^{-2} \mathrm{~s}^{-1}\right)\end{array}$ & $\begin{array}{c}\text { BUSSM } \\
(3 \sigma)\end{array}$ & $\begin{array}{c}\operatorname{TSSM}^{\dagger} \\
(1 \sigma)\end{array}$ & $\begin{array}{c}\text { Bahcall et al. } \\
1992 \\
\left(\mathrm{w} /{ }^{4} \mathrm{He}\right. \\
\text { diffusion, } 3 \sigma)\end{array}$ \\
\hline$p p$ & $600(1 \pm 0.02)$ & $598(1 \pm 0.03)$ & $600(1 \pm 0.02)$ \\
\hline pep & $1.4(1 \pm 0.05)$ & 1.3 & $1.43(1 \pm 0.04)$ \\
\hline${ }^{7} \mathrm{Be}$ & $47(1 \pm 0.15)$ & 41.8 & $48.9(1 \pm 0.18)$ \\
\hline${ }^{8} \mathrm{~B}$ & $5.8(1 \pm 0.37)$ & $3.83(1 \pm 0.27)$ & $5.69(1 \pm 0.43)$ \\
\hline${ }^{13} \mathrm{~N}$ & $6.1(1 \pm 0.50)$ & 6.27 & $4.92(1 \pm 0.51)$ \\
\hline${ }^{15} \mathrm{O}$ & $5.2(1 \pm 0.58)$ & 5.6 & $4.26(1 \pm 0.58)$ \\
\hline
\end{tabular}

- Before the correction on TSSM. See ref. 7

Two currently uncertain input parameters critically affect the ${ }^{8} \mathbf{B}$ neutrino flux $[1,2]:(1)$ the uncertainty in the extrapolated cross section for the ${ }^{7} \mathrm{Be}(p, \gamma)^{8} \mathrm{~B}$ reaction; (2) the primordial heavy element abundance $Z$ and its effect on calculated radiative opacity. The ${ }^{7} \mathrm{Be}(p, \gamma)^{8} \mathrm{~B}$ reaction produces ${ }^{8} \mathrm{~B}$ which emits a neutrino as a decay product. Therefore its rate linearly affects the calculated ${ }^{8} \mathrm{~B}$ neutrino flux. The different values adopted in BUSSM and TSSM reflect the large spread in choosing this parameter. A higher $Z$ and a higher opacity increase the temperature gradient and therefore lead to a higher core temperature $T_{c}$ inside the sun. It was shown by Bahcall and Ulrich that the ${ }^{8} \mathrm{~B}$ neutrino flux $\phi\left({ }^{8} \mathrm{~B}\right) \propto T_{c}^{18}$ $[1,2]$. Therefore a tiny difference in $T_{c}$ will cause a significant difference in ${ }^{8} \mathbf{B}$ neutrino flux.

The uncertainty in the ${ }^{7} \mathrm{Be}$ neutrino flux comes primarily from its temperature dependence. Its flux $\phi\left({ }^{7} \mathrm{Be}\right) \propto T_{c}^{8}[1,2]$.
Since the ${ }^{7} \mathrm{Be}$ neutrino is predicted to contribute considerably in the chlorine and gallium experiments (see table 2), its uncertainty also needs to be considered carefully.

The CNO neutrinos have even larger relative uncertainties than the ${ }^{8} \mathrm{~B}$ neutrinos due to their more sensitive $T_{\mathrm{c}}$ dependence $[1,2]$. But since they are minor contributions in the experiments, their uncertainties don't play a major role in the solar neutrino problem.

The $p p$ neutrinos are the most abundant and the least uncertain neutrino source among the solar neutrinos. Due to the constraints of the solar model observables, its flux $\phi(p p) \propto$ $T_{c}^{-1.2}[1,2]$. It consists of more than half of the neutrino events that the gallium experiments can see. An observed rate lower than the predicted $p p$ neutrino contribution in the gallium experiments will definitely indicate that the $p p$ neutrinos are depleted, and new neutrino physics would need to be introduced. From energetic considerations, regardless of the detail of solar models, the requirement of reproducing the observed solar luminosity through nuclear reactions will yield a minimal neutrino flux of about $80 \mathrm{SNU}$ in the gallium experiments (in which case only $p p$ and pep neutrinos are produced) [30]. Therefore, an observed solar neutrino flux significantly lower than $80 \mathrm{SNU}$ in the gallium experiments would be a smoking gun of new neutrino physics. So far, neither of the two gallium experiments yields a neutrino flux significantly lower than $80 \mathrm{SNU}$ with confidence. Therefore the evidence of new neutrino physics is not compelling. Furthermore, because of the large uncertainties in the ${ }^{8} \mathrm{~B}$ neutrinos, the observed deficit in the Kamiokande experiment can be easily avoided by modifying the standard solar model within a reasonable uncertainty range and a ${ }^{37} \mathrm{Cl}$ result as low as $\sim 4 \mathrm{SNU}$ might not be out of the question. However, ${ }^{37} \mathrm{Cl}$ rates below $4 \mathrm{SNU}$ are definitely problematic for any pure solar model input parameter variation. Let us now look in more detail at the crucial solar model input parameters.

The reaction rate of ${ }^{7} \mathrm{Be}(p, \gamma)^{8} \mathrm{~B}$ that affects the ${ }^{8} \mathrm{~B}$ neutrino flux is acquired by extrapolating the measured cross section at high energy (110 keV to $4000 \mathrm{keV})$ to the astrophysical interesting energy of $\sim 20 \mathrm{keV}$. Its true uncertainty is difficult to extract. Experiments at relatively high energy were done and were improved gradually over time. The extrapolation calculation has also been improved over the course of more than two decades. BUSSM (1988) used $S(0)=24.3 \mathrm{eV} \cdot \mathrm{b}$, based upon the extrapolation method of Tombrello (1965) [31], which only included the s-wave extrapolation. TSSM adopted $S(0)=$ $21 \pm 3 \mathrm{eV} \cdot \mathrm{b}$ from Barker's extrapolation including both s-wave and d-wave [32]. The most recent calculation including both $s$-wave and d-wave done by Johnson et al. gives an average $S(0)$ for six experiments $S(0)=22.4 \pm 1.0 \mathrm{eV} \cdot \mathrm{b}$ [33], which is adopted by Bahcall et al. in their 1992 solar model. Among the six experiments, two experiments done by Kavanagh [34] in 1969 and Filippone [35] in 1983 did measurements at low 
energy below $430 \mathrm{keV}$, which is crucial to the extrapolation [33]. These two experiments disagree with each other at low energy at about the $2 \sigma$ level. Therefore instead of taking the average value of the six experiments or the two more satisfactory experiments, it may be more appropriate to take either of the values of the Kavanagh experiment or the Filippone experiment, which are $25.2 \pm 2.4 \mathrm{eV} \cdot \mathrm{b}$ and $20.2 \pm 2.3 \mathrm{eV} \cdot \mathrm{b}$ respectively [33]. If the value of the Filippone experiment, which is more recent and more carefully described, is taken, the ${ }^{8} \mathrm{~B}$ neutrino flux in BUSSM will decrease by $17 \%$, from $6.1 \mathrm{SNU}$ to 5.1 SNU for the chlorine experiment [19]. The total flux predicted by BUSSM is then $6.9 \mathrm{SNU}$. The ${ }^{8} \mathrm{~B}$ neutrino flux in the improved Bahcall model with helium diffusion will decrease by $10 \%$, giving a total flux of $7.4 \mathrm{SNU}$. The TSSM prediction will also decrease from a total of $6.4 \mathrm{SNU}$ to $6.2 \mathrm{SNU}$. Besides the fact that the ${ }^{8} \mathrm{~B}$ neutrino flux will be diminished, its uncertainty coming from the uncertainty of this reaction rate will be directly inferred from the error of the rate, instead of estimated from averaging over different values. The total uncertainty in ${ }^{8} \mathrm{~B}$ neutrino flux thereby increases from $37 \%$ to $44 \%$ for the BUSSM, and from $43 \%$ to $48 \%$ for the improved Bahcall model with helium diffusion, which further obscures the neutrino deficit [36]. But for TSSM, the uncertainty in ${ }^{8} \mathrm{~B}$ neutrino flux decreases from $29 \%(1 \sigma)$ to $27 \%(1 \sigma)[36]$. Obviously, additional low energy ${ }^{7} \mathrm{Be}(p, \gamma)^{8} \mathrm{~B}$ measurements are needed.

Further reductions in neutrino fluxes can be achieved by lowering the $T_{c}$. Various non-standard solar models [2] have been proposed to yield a lower $T_{c}$ and hence lower neutrino flux relative to the standard solar models. For example, in the WIMPs (Weakly Interacting Massive Particles) model, the WIMPs captured by the Sun can transport the nuclear energy produced at the core and lead to a smaller temperature gradient and a lower $T_{c}$; in the strong magnetic field model, a magnetic field of $10^{9}$ Gauss at the center of the Sun will provide part of the pressure to support the Sun, and therefore lower the $T_{c}$; in the mixing model, the Sun is mixed up through turbulent diffusion or other hydrodynamical effects and a lower $T_{c}$ is achieved. These models are not as successful as the standard solar models in fitting standard solar observational properties, and are rather ad hoc [2]. In the context of standard solar models, a lower $T_{c}$ can be achieved by lowering the heavy element abundance $Z$ and the opacity. The solar element abundances are obtained from the spectrometry of the solar suface and the measurements from the meteorites, assuming the abundances there are primordial [37]. For most of the elements, the two types of measurements agree within $9 \%$. But for iron, which contributes $\sim 20 \%$ of the opacity, the two measurements are about $4 \sigma$ away from each other. The iron to hydrogen abundance ratio from the photospheric measurement is $4.68( \pm 0.33) \times 10^{-5}$, while the meteorite measurement gives $3.25( \pm 0.075) \times 10^{-5}$. If the lower value of the meteorite measurement is used, the contribution to the opacity from the iron will be substantially reduced, which in turn lowers the core temperature and the neutrino flux. Bahcall et al. found that the improved Bahcall model (1992) without helium diffusion yielded 8.5 SNU for the chlorine experiment when the photospheric value was used, and 7.2 SNU when the meteoritic value was used [16]. Furthermore, Marx and Dearborn [38] have discussed further Fe depletion in the opacity by proposing Fe-clustering due to some unknown mechanism. Such clustering could further reduce the solar opacity and thus $T_{c}$ (but would still not yield a ${ }^{37} \mathrm{Cl}$ result below $3 \mathrm{SNU}$ ).

In an attempt to construct a low flux solar model that is consistent with the experiments, we have evolved the solar code of Dearborn. We adopt $S(0)=20.2 \mathrm{eV} \cdot \mathrm{b}$ for the reaction ${ }^{7} \mathrm{Be}(p, \gamma){ }^{8} \mathrm{~B}$, and BUSSM values [1] for other rates. We also adopt the OPAL opacity table with an Anders and Grevesse meteoritic mixture [37]. By choosing $Z=0.015, X=0.723$, our model yields a neutrino flux of $4.5 \mathrm{SNU}$ for the chlorine experiment. The ${ }^{8} \mathrm{~B}$ contribution is $3.3 \mathrm{SNU}$, a $46 \%$ reduction with respect to that of BUSSM. The ${ }^{7} \mathrm{Be}$ neutrinos contribute 0.9 SNU. The rest 0.3 SNU is almost entirely from CNO neutrinos. The predicted event rate for the two Kamiokande experiments is 0.54 of the BUSSM prediction. The prediction for the gallium experiments is about 114 SNU. These predictions agree with the Kamiokande results very well, agree with GALLEX within $1.5 \sigma$, and agree with SAGE result within $2.5 \sigma$. Compared with the chlorine result, given an theoretical uncertainty of $\sim 15 \%(1 \sigma)$, our prediction is no more than $3 \sigma$ away. (But one should be careful here because at low fluxes the theoretical uncertainty goes non-linear [18]. It is very difficult to construct a solar model with less than 3 SNU for the chlorine experiment.) Therefore given both the theoretical uncertainties and the experimental uncertainties, it is conceivable that there could be no significant conflict between the experiments and such a minimized solar model. The core temperature $T_{c}$ of this model is $1.545 \times 10^{7} \mathrm{~K}$, compared to $1.564 \times 10^{7} \mathrm{~K}$ of BUSSM and $1.551 \times 10^{7} \mathrm{~K}$ of TSSM (table 3 ). Obviously the heavy element abundance adopted is lower than those of other standard solar models. But it is only $2.4 \sigma$ below the heavy element abundance obtained by Anders and Grevesse based on the meteoritic measurement ( $Z=0.01886 \pm 0.0016)$ or $2.7 \sigma$ below the abundance based on the solar photospheric measurement $(Z=0.01941 \pm 0.0016)[37]$. These modifications do not affect the agreement between standard solar models and astronomical observations.

\section{The Current Status of the Solar Neutrino Problem}

It has been accurately argued by Bahcall and Bethe [39] that the disagreement between the results of the ${ }^{37} \mathrm{Cl}$ experiment $(28 \% \pm 4 \%$ the BUSSM prediction) and the Kamiokande II $(46 \% \pm 8 \%$ the BUSSM prediction) implies neutrino oscilla- 
tions. Obviously, assuming the full validity of both experiments, if nothing happens after the solar neutrinos are produced, the ${ }^{8} \mathrm{~B}$ neutrino contribution to the ${ }^{37} \mathrm{Cl}$ experiment is at most $2.2 \pm 0.3 \mathrm{SNU}$, corresponding to $36 \% \pm 5 \%$ the BUSSM prediction. It barely agrees with the Kamiokande II result (the problem is even more severe for the Kamiokande III result). But if $v_{e}$ oscillates into $v_{\mu}$ or $v_{\tau}$, the remaining $64 \%$ or so now changed into $v_{\mu}$ or $v_{\tau}$ will interact with the electrons via neutral currents, with $\sigma\left(v_{\mu \text { or } \mathrm{r}}-\mathrm{e}\right) / \sigma\left(v_{c}-\mathrm{e}\right)=1 / 6 \sim 1 / 7$ [2]. So the total rate inferred from the Homestake experiment for the Kamiokande II would be $36 \%+64 \% \times 1 / 6 \approx 46 \%$ the BUSSM prediction, which agrees with the Kamiokande II measurement extremely well.

Because the ${ }^{37} \mathrm{Cl}$ experiment observes both the ${ }^{8} \mathrm{~B}$ neutrinos and the ${ }^{7} \mathrm{Be}$ neutrinos, and the Kamiokande II experiment detects only the ${ }^{8} \mathrm{~B}$ neutrinos, the above agreement implies that nearly no ${ }^{7} \mathrm{Be}$ neutrinos are observed in the ${ }^{37} \mathrm{Cl}$ experiment. If the ${ }^{7} \mathrm{Be}$ neutrinos are entirely depleted and the ${ }^{8} \mathrm{~B}$ neutrinos are depleted by about a half to twc-thirds, as implied by the Kamiokande and Homestake experiments, the gallium experimental yield would be $\sim 90$ SNU, provided no $p p$ neutrino reduction, which would be in good agreement with the GALLEX and SAGE results. In fact, without resorting to any new physics, if the chlorine experiment yield is smaller or equal to the yield of the Kamiokande experiments with respect to the prediction, the ${ }^{7} \mathrm{Be}$ neutrinos have been entirely missing.

No known astrophysical solution can explain a higher ${ }^{7} \mathrm{Be}$ neutrino reduction than ${ }^{8} \mathrm{~B}$ neutrino reduction relative to the standard solar model prediction. A lower central temperature in the Sun apparently doesn't help because the ${ }^{8} \mathrm{~B}$ neutrinos are more temperature sensitive than the ${ }^{7} \mathrm{Be}$ neutrinos as mentioned before. In terms of nuclear physics, the ${ }^{7} \mathrm{Be}$ neutrinos and the ${ }^{8} \mathrm{~B}$ neutrinos are produced by two different channels of the ${ }^{7} \mathrm{Be}$ nuclear reaction $\left({ }^{7} \mathrm{Be}\left(\mathrm{e}, v_{e}\right)^{7} \mathrm{Li}\right.$ for ${ }^{7} \mathrm{Be}$ neutrinos; ${ }^{7} \mathrm{Be}(p, \gamma){ }^{8} \mathrm{~B}$ and subsequent ${ }^{8} \mathrm{~B} \beta$-decay for ${ }^{8} \mathrm{~B}$ neutrinos) [2]. If one suppresses the ${ }^{7} \mathrm{Be}$ neutrinos by sup pressing the overall ${ }^{7} \mathrm{Be}$ production, the ${ }^{8} \mathrm{~B}$ neutrinos will be reduced by the same factor. If one suppresses the branching ratio that produces ${ }^{7} \mathrm{Be}$ neutrinos, ${ }^{8} \mathrm{~B}$ neutrino flux will increase, opposite to the experimental implication. Therefore, the current experimental results, at their face value, suggest new physics, if not in particle physics, then in the more fundamental nuclear physics. Thus once Bahcall and Bethe assumed the validity of both the Homestake and Kamiokande experiments, they were led to new physics.

Since the two most controversial experiments, the Homestake and SAGE, happen to yield the largest reductions with respect to the standard solar models, it is interesting to see how the situation of astrophysical solution vs. new neutrino physics could change if, say, unanticipated systematic errors did exist in either or both of these experiments. For example, in the chlorine experiment, if only runs after the pump failures are considered, which yield an unweighted average of $2.8+0.6$ $\mathrm{SNU}$, the above ${ }^{7} \mathrm{Be}$ neutrino vs. ${ }^{8} \mathrm{~B}$ neutrino argument for new physics may not be strong when the experimental and theoretical uncertainties are fully taken into account. For SAGE, one may argue that it might be safer to use only their latest runs, which yield $85_{-32}^{+22} \pm 20 \mathrm{SNU}$.

In order to show the sensitivity of the MSW solution to the experimental results relative to a modified solar model in being able to solve the solar neutrino deficit, we treat the solar core temperature $T_{c}$, the neutrino mixing parameters $\Delta m^{2}$ and $\sin ^{2} 20 / \cos 20$ (we assume a two-family mixing between $v_{c}$ and non-sterile $v_{x}$ ) as three free parameters and follow Bludman et al. to do a $\chi^{2}$ test to see how well the experimental data can be fit by adjusting these parameters [40]. The MSW mixing is represented by parameters $\Delta m^{2}$ and $\sin ^{2} 20 / \cos 20$. The astrophysical solutions are represented by lower $T_{c}$ 's with respect to $T_{c}$ (BUSSM), where the neutrino fluxes are assumed to follow the $T_{c}$ dependence as calculated by Bahcall and Ulrich in ref. 1. Generally, the core temperature can be as low as $\sim 1.54 \times 10^{7} \mathrm{~K}\left(\sim 0.98 T_{c}\right.$ (BUSSM) $)$ in the uncertainty range of standard solar models [2]. Additional $T_{c}$ reductions may be achieved by nonstandard solar models. But below $0.98 T_{c}$ (BUSSM), the temperature dependence of different neutrino sources may vary. Therefore, our calculation is for illustrative purpose when $T_{c}$ goes beyond the standard solar model range. We have replaced the ${ }^{7} \mathrm{Be}(p, \gamma)^{8} \mathrm{~B}$ rate in BUSSM with the rate calculated by Johnson from Filippone's experiment. So when $T_{c}=T_{c}$ (BUSSM), the standard solar model yields $6.9 \mathrm{SNU}$ instead of $7.9 \mathrm{SNU}$.

The MSW solution with astrophysical uncertainties, for both the two-family and three-family mixing, has been discussed by Shi, Schramm and Bahcall [11]. Fig. 3 shows the two family MSW solution and the three family MSW solution for 1000 solar models [1] whose input parameters are chosen within the theoretical uncertainty range via a Monte Carlo method. In the three-family case, we assume a mass hierachy between three generations and a large mixing angle between $v_{e}$ and $v_{\tau} \phi=0.5 \mathrm{rad}$. Small $v_{e}-v_{\tau}$ mixing three-family MSW solutions are intermediate cases between the two shown. The conclusion is that the three-family MSW solution doesn't alter the basic picture of the two-family MSW solution. Therefore we only consider the two-family MSW solution in our test. By calculating the $\chi^{2}$ of the two-family MSW solution and low $T_{c}$ astrophysical solutions for the current solar neutrino experiments, we can find out how well they fit the experimental data. In the test, we have included both the experimental errors and the theoretical errors coming from the $S(0)$ factor of the reaction ${ }^{7} \mathrm{Be}(p, \gamma)^{8} \mathrm{~B}$. The theoretical uncertainties of $Z$ and opacities have been represented by different choices of $T_{c}$. We obtain $\chi^{2}$ for the Homestake experiment, the Kamiokande II, the Kamiokande III, GALLEX and SAGE. In order to show how the Homestake experiment and SAGE can affect our re- 


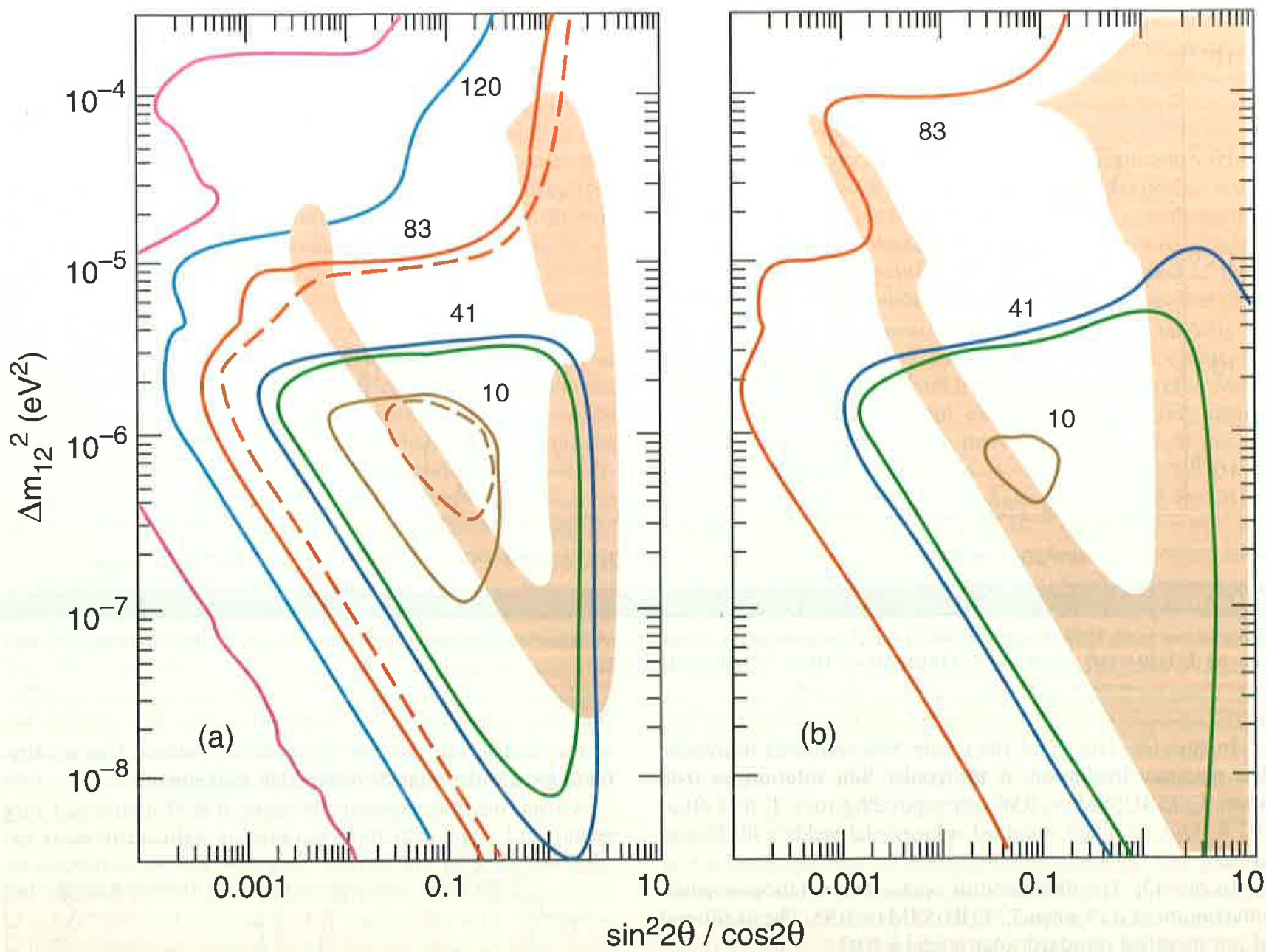

Assuming two-family MSW mixing, the shaded region is the MSW solution allowed by both the ${ }^{37} \mathrm{Cl}$ experiment and the Kamiokanda II experiment at $95 \% \mathrm{C}$.L., using Monte Carlo simulations of the theoretical uncertainties in 1000 solar models. The iso-SNU bands for the ${ }^{11}$ Ga experiment are also shown in solid lines (GALLEX gives $83 \pm 21(1 \sigma)$ SNU). (a) two-family mixing; (b) three-family mixing with $\phi=0.5 \mathrm{rad}$ ( $\phi$ is the mixing angle between $v_{c}$ and $v_{r}$ ).

sult, we have done the test for three cases: (1) the experimental result as shown in table 1; (2) same as case (1) except a higher Homestake rate $2.8 \pm 0.6$ SNU (which is the unweighted average over the period after the pump failures); and (3) same as (1) except a SAGE rate of $85_{-32}^{+22} \pm 20 \mathrm{SNU}$ (which is the average rate of their recent less controversial 6 runs).

In case (1), the MSW solution with varying core temperatures has a minimal $\chi^{2}$ of 0.89 , when $T_{c} / T_{c}$ (BUSSM) $=1.04$. Figure 4 shows the $\chi^{2}$ contours with C.L. of $90 \%$ and $95 \%$ respectively in the MSW mixing parameter space for different
$T_{c} / T_{c}$ (BUSSM)'s. Two MSW solutions exist: the diagonal solution and the vertical solution, which agrees with the usual MSW analysis. When assuming the BUSSM, the diagonal solution is more favored than the vertical one. But as $T_{c}$ increases, the likelihood of the vertical region also increases. For comparison, the likelihood of the astrophysical solutions without neutrino mixing has a maximum of 0.04 when $T_{c} / T_{c}(\mathrm{BUSSM})=$ 0.95 . The likelihood of the model discussed at the end of Section 3 for the five experiments is 0.006 , which is ruled out at $99 \%$ C. L. 


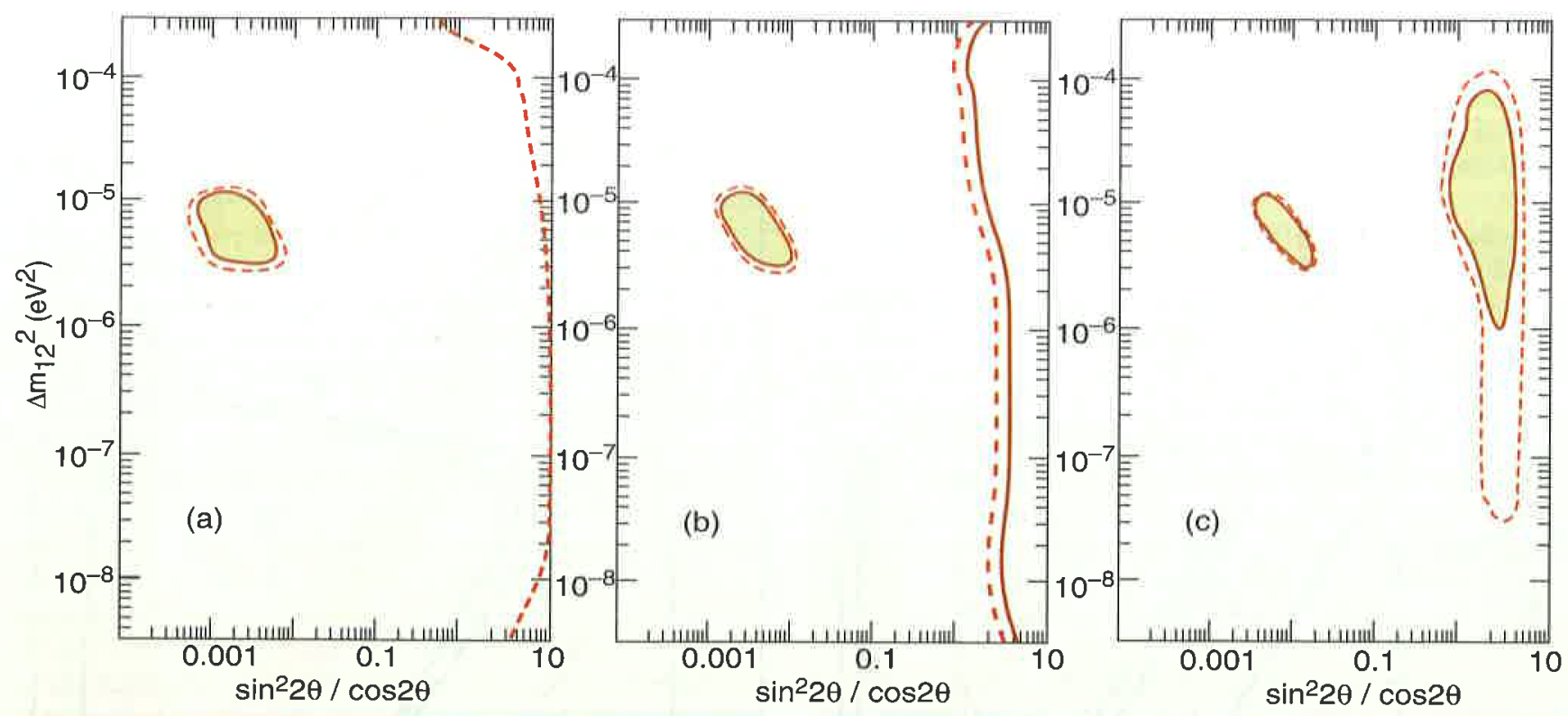

\section{FIGURD 4}

Assuming two-family MSW mixing and case (1), the $\chi^{2}$ contours for the five running solar neutrino experiments. The solid line: $90 \%$ C.L.; the short-dashed line: $95 \%$ C.L.. (a) $T_{c} / T_{c}$ (BUSSM) $=0.98 ;$ (b) $T_{c} / T_{c}$ (BUSSM) $=1.00 ;$ (c) $T_{c} / T_{c}$ (BUSSM) $=1.02$.

In case (2), The $\chi^{2}$ of the cooler Sun solutions decreases. The maximal likelihood of the cooler Sun solutions is 0.08 when $T_{c} / T_{c}($ BUSSM $) \approx 0.96$, corresponding to a $T_{c}$ of $1.50 \times$ $10^{7} \mathrm{~K}$. Our modified standard solar model yields a likelihood of 0.04 .

In case (3), The likelihood of cooler Sun solutions reaches a maximum of 0.23 when $T_{c} / T_{c}($ BUSSM $) \approx 0.95$. The likelihood of our modified standard solar model is 0.03 .

If one uses the higher yields for both the Homestake experiment and. SAGE as in cases (2) and (3), the cooler Sun solutions can only be ruled out at $57 \%$ C.L. Similarly, our modified solar model is only ruled out at $80 \%$ C.L.

Figure 5 shows the $\chi^{2}$ contours of the MSW solutions for case (2) and case (3), and $T_{c} / T_{c}(B U S S M)=1$ only. They are not very different from that of case (1).

We have also performed Bayesian Inference [41] which prefers models with fewer parameters to compare the MSW solution with BUSSM with the low $T_{c}$ solutions. In case (1), the MSW solution with BUSSM is favored over the low $T_{c}$ solutions by $\mathcal{O}\left(10^{2}\right)$ to 1 ; in case (2), the two models are nearly equally probable; in case (3), the MSW solution with BUSSM is still more favored by $\mathcal{O}(10)$ to 1 . In the case which both the Homestake and SAGE experiments give high yields as in case (2) and (3) respectively, the cooler Sun solution may be slightly more favored. The Bayesian analysis apparently depends quantitatively on the prior probability assigned to each parameters of the models, but the above conclusion remains true qualitatively for a wide range of reasonable assignments.

Given only five experimental data, it is of course not very meaningful to fit with three parameters. Obviously more ex-

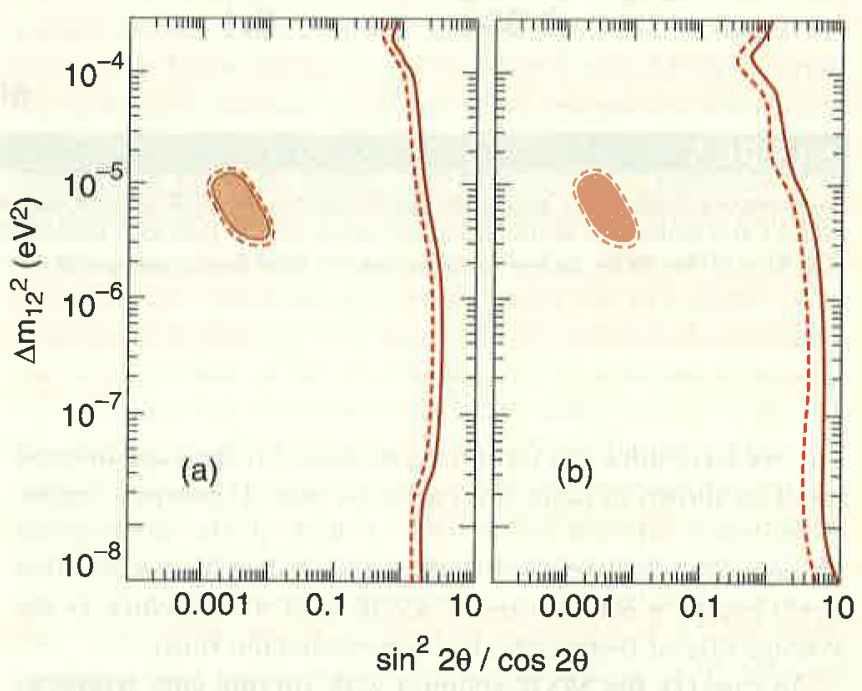

\section{FIGURE 5}

The same graph as fig. 4 for case (2) and (3), with $T_{c} / T_{c}($ BUSSM) $=1.00$ only. (a) case (2); (b) case (3). 
periments need to be done to decisively rule out any of the alternative solutions. But if the current solar neutrino experimental results are taken at face value as in table 1 , we find that the astrophysical solutions may be disfavored, especially for standard solar models. But if either the chlorine result or the gallium result is higher than listed in table 1, the cooler Sun solutions may fit the data fairly well. Therefore the astrophysical solution to the solar neutrino problem may, given the current experimental situation, remain plausible and viable.

\section{Future Prospects}

In order to resolve the different solutions, in addition to the calibrations needed to be done for current experiments, information from next generation experiments seems to be necessary. The additional information should include: (1) the spectral shape of the incoming solar neutrinos; (2) the statistically significant data on the neutral current component in the scattering events; (3) the diurnal and seasonal variations of signals; and (4) limits on possible antineutrino components in the solar neutrino flux.

The spectral shape of the solar neutrino flux provides one possible way to distinguish the new neutrino physics solution from the astrophysical solution. Different solutions generate different neutrino spectrums at the detectors. The astrophysical solution will reduce the ${ }^{8} \mathrm{~B}$ neutrino spectrum uniformly with respect to the BUSSM. Other neutrino fluxes remain more or less the same. In the MSW solution, the horizonal region $\left(\Delta m^{2} \sim 10^{-4} \mathrm{eV}^{2}\right)$ which has been ruled out so far would show almost complete depletion at high energies and nearly no depletion at low energies; among the two regions that are allowed by the current experiments, the diagonal region $\left(2 \times 10^{-6} \mathrm{eV}^{2}<\Delta m^{2}<10^{-4} \mathrm{eV}^{2}, \Delta m^{2} \sin ^{2} 2 \theta \sim 10^{-7} \mathrm{eV}^{2}\right)$ has a larger depletion rate at low energies than at high energies, while the vertical solution $\left(10^{-8} \mathrm{eV}^{2}<\Delta m^{2}<10^{-4} \mathrm{eV}^{2}\right.$, $\sin ^{2} 2 \theta>0.5$ ) yields a uniform depletion over the whole energy range. The long wave length vacuum mixing solution $\left(\Delta m^{2} \sim\right.$ $10^{-10} \mathrm{eV}^{2}, \sin ^{2} 2 \theta>0.7$ ), also shows a distortion in spectrum with respect to the BUSSM, with the distortion depending on the detail of the mixing parameters [9]. The spin-flavor precession neutrino magnetic moment solution $\left(\mu_{y} \sim 10^{-11} \mu_{B}\right)$ is similar to the MSW solution $[13,14]$, that has three regions exhibiting different spectrums. The neutrino decay solution, in which the solar neutrinos decay on their way to the earth, will have a lower depletion rate at higher energies because the lifetime of neutrinos is proportional to their energies [15].

So far, only the Kamiokande experiments have spectral resolutions of $\sim 22 \%$ at $10 \mathrm{MeV}$ [20]. But because of its low statistics, the spectrum obtained by the Kamiokande II experiment only rules out a small region of the horizontal branch of the MSW solution which can also be ruled out by the combination of the ${ }^{37} \mathrm{Cl}$ experiment and the Kamiokande II ex- periment. The spectrum shows no appreciable distortion with respect to the BUSSM, and is consistent with the diagonal and the vertical MSW solutions, as well as the astrophysical solution, and all other solutions that have a uniform depletion over the ${ }^{8} \mathrm{~B}$ neutrino spectrum above $8 \mathrm{MeV}$.

The neutral current interaction is indiscriminate to different flavors. Therefore its component of the scattering events includes contributions from $v_{e}, v_{\mu}$ and $v_{\tau}$. The $v_{e}$ contribution, however, can be calculated from charged current events, which only involve $v_{e}$. Any neutral current events in excess of the $v_{e}$ contribution clearly implies the presence of neutrinos of other flavors, that the solar neutrinos oscillate into. So far only the Kamiokande experiments can see neutral current events, but they cannot distinguish them from the charged current events. Though the charged current events can be roughly infered from the chlorine experiment, there is no independent method to obtain evidence of oscillation.

The diurnal variation is a unique signature of the MSW matter mixing. For the region with $10^{-6} \mathrm{eV}^{2}<\Delta m^{2}<10^{-5} \mathrm{eV}^{2}$ on the MSW parameter space, resonances could occur when neutrinos propagate through the earth. Such resonances convert $v_{e}$ to other flavors and vice versa, which is the so-called earth regeneration effect [42]. If the $v_{e}$ component in the solar neutrinos is not $1 / 2$ when they reach the earth, such a regeneration effect will make a difference between daytime event rates and night time event rates in direct counting experiments. It will also cause an extra seasonal variation besides that from the simple $1 / R^{2}$ effect (where $R$ is the distance between the earth and the Sun). The long wave length vacuum oscillations also show the extra seasonal variation due to the sensitivity of the depletion rate to the distance between the Sun and the earth. But there is no day-night variation in this case. For astrophysical solutions, however, nothing could happen to neutrinos on their way to the earth. Therefore no diurnal variations or seasonal variations besides that from the $1 / R^{2}$ effect could occur. Such variations have been searched for in the Kamiokande II experiment. The result is (night events day events) $/($ night events + day events $)=-0.08 \pm 0.11 \pm 0.03$, which is consistent with no variation, and rules out the area $2 \times 10^{-6} \mathrm{eV}^{2}<\Delta m^{2}<10^{-5} \mathrm{eV}^{2}$ on the vertical region of the MSW solution [43]. Obviously more statistics are needed to draw a more decisive conclusion on the time variations.

The existance of an antineutrino component in the solar neutrino flux is a unique signature of majorana neutrinos with magnetic moments. If electron neutrinos are majorana neutrinos and possess magnetic moments, left-handed solar neutrinos can be converted to right-handed antineutrinos in the solar magnetic field [3]. Current solar neutrino experiments are not sensitive to the signature of solar antineutrinos.

If solar neutrinos oscillate into sterile neutrinos, the neutral current test cannot resolve neutrino oscillations from astrophysical solutions. Spectral distortions and time variations are 
then tests for this resolution. But since a large portion of the mixing parameter space of the neutrino oscillation solutions (including the MSW mixing, the vacuum mixing and the spinflavor precession) yields no appreciable spectral distortion and time variation, the currently available tests cannot distinguish them from astrophysical solutions. There could be constraints from oscillation experiments, cosmology and astrophysics. For example, the constraint on extra neutrino species from the Big Bang Nucleosynthesis prohibits large angle MSW mixing between $v_{e}$ and sterile neutrinos [44]. But the small mixing angle oscillation between $v_{e}$ and sterile neutrinos seems very hard to test at present

Solar neutrino experiments of the next generation could provide the additional information to resolve the solar neutrino problem. Right now four experiments have been approved for construction or are proposed for construction: the Super Kamiokande, SNO (Sudbury Neutrino Observatory), Borexino, and the Iodine experiment. They are all direct counting real-time experiments except the Iondine experiment, which is radiochemical. A summary of the four experiments is in Table 5 [21].

The Super Kamiokande is an upgraded version of the, Kamiokande II. With a fiducial mass about 30 times larger than the Kamiokande II, and a lower threshold, the Super Kamiokande is estimated to have an event rate $\sim 50$ times higher than the Kamiokande II. It will provide significantly better statistics in terms of determining the neutrino flux, the spectrum shape and any diurnal or seasonal variation.

The SNO project utilizes $1 \mathrm{kton}$ of heavy water $\left(\mathrm{D}_{2} \mathrm{O}\right)$ to interact with solar neutrinos. It has the following reactions: (1) $v_{e}+\mathrm{d} \rightarrow \mathrm{p}+\mathrm{p}+e^{-}$, via the charged current; (2) v-e elastic scattering via both the charged and the neutral currents; and (3) $v+\mathrm{d} \rightarrow v+\mathrm{p}+\mathrm{n}$ via the neutral current only. Reaction (2) and (3) can see not only $v_{e}$, but also other flavors of neutrinos. By comparing the neutral current events and the charged current events measured from reactions (1), (2) and (3), neutrino oscillations can then be tested. SNO can also detect the existance of solar antineutrinos via the reaction $\bar{v}_{e}+\mathrm{d} \rightarrow e^{+}+n+n$, which provides unique evidence for the majorana neutrino magnetic moment solution. SNO also has better spectral resolution and time variation resolution than current experiments because of the higher energy resolution and event rate.

Borexino [45], the first phase of the Borex program, exploits liquid scintillator (a mixture of about $85 \%$ of trimethylborate and $15 \%$ aromatic liquid such as pseudocumene) as its target. Borexino uses the same neutrino-electron scattering reaction to observe solar neutrinos, as in the Kamiokande experiment. But unlike the Kamiokande and SNO, where the Cerenkov radiations of the recoil electrons are used as signals, Borexino uses the scintillator light of the recoil electrons to determine their spectrum. Because of the ultrapurity of the scintillator that is available, Borexino sees mainly the low-energy

Table 5

Future solar neutrino experiments

\begin{tabular}{|c|c|c|}
\hline & Super Kamiokande & Borexino \\
\hline $\begin{array}{l}\text { Detector material } \\
\text { Fiducial mass }\end{array}$ & $\begin{array}{c}\mathrm{D}_{2} \mathrm{O} \\
1 \text { kton }\end{array}$ & $\begin{array}{cc}\text { Liquid Scintillator } & \mathrm{NaI} \\
100 \text { ton } & 1 \mathrm{kton}\end{array}$ \\
\hline Reaction & $\begin{array}{c}v_{e}+\mathrm{d} \rightarrow \mathrm{p}+\mathrm{p}+\mathrm{e}^{-} \\
v-\mathrm{e} \text { scattering } \\
v+\mathrm{d} \rightarrow v+\mathrm{p}+\mathrm{n} \\
v_{e}, v_{\mu}, v_{\tau}, \bar{v}\end{array}$ & $\begin{array}{cc}\nu \text {-e scattering } & v_{e}+{ }^{127} \mathrm{I} \rightarrow \mathrm{e}^{-}+{ }^{127} \mathrm{Xe} \\
v_{e}, v_{\mu}, v_{\tau}, \bar{v} & v_{e} \text { only }\end{array}$ \\
\hline Threshold & $\sim 5 \mathrm{MeV}$ & $0.789 \mathrm{MeV}$ \\
\hline Type & $\begin{array}{c}\text { Direct Counting } \\
\text { Real Time } \\
\text { Spectroscopic } \\
\text { Directional }\end{array}$ & $\begin{array}{c}\text { Radiochemical } \\
\text { Time Integral } \\
\text { Energy Integral } \\
\text { Non-directional }\end{array}$ \\
\hline $\begin{array}{l}\text { Energy resolution } \\
(10 \mathrm{MeV}) \\
(1 \mathrm{MeV})\end{array}$ & $\begin{array}{cc}14 \% & 14 \% \\
- & -\end{array}$ & $\begin{array}{l}4 \% \\
7.5 \%\end{array}$ \\
\hline $\begin{array}{l}\text { Event rate } \\
\text { estimated by BUSSM }\end{array}$ & $\sim 30 /$ day & $\sim 50 /$ day \\
\hline
\end{tabular}


high-flux ${ }^{7} \mathrm{Be}$ neutrinos with a rate of up to 50 events/day, which is unique among the solar neutrino experiments. Comparing the low energy ${ }^{7} \mathrm{Be}$ neutrino flux observed by Borexino with the high energy ${ }^{8} \mathrm{~B}$ neutrino flux observed by other experiments, one may have a good picture of the depletion rates at different part of the spectrum, and a possible resolution of different solutions. In terms of MSW solutions, it has been shown that the currently allowed diagonal solution and the vertical solution may yield different ${ }^{7} \mathrm{Be}$ neutrino fluxes [11]. Thus the Borexino experiment may yield stricter constraints on the MSW solution. The high event rates also enable Borexino to have a high sensitivity in observing the recoil electron spectrum, the time variation of the flux, that is essential in resolving the solar neutrino problem. Because of the low energy of ${ }^{7} \mathrm{Be}$ neutrinos, the region on the MSW parameter space that would exhibit large time variation in Borexino in Borexino is quite different from that in the Kamiokande experiments, namely it is at the lower corner of the MSW triangle [45]. Therefore this part of the solution can be constained by the time variation measurement of Borexino. Finally, Borexino has sensitivity to solar antineutrinos by detecting $e^{+}$produced by the reaction $\bar{v}_{e}+p \rightarrow e^{+}+n$.

The Iodine experiment [46] is very similar to the chlorine experiment. Via the reaction

$$
v_{e}+{ }^{127} \mathrm{I} \rightarrow e^{-}+{ }^{127} \mathrm{Xe}, \quad E_{\mathrm{lh}}=0.789 \mathrm{MeV},
$$

solar neutrinos are absorbed by the $\mathrm{NaI}$ desolved in water. ${ }^{127} \mathrm{Xe}$ is extracted in the same way as ${ }^{37} \mathrm{Ar}$ in the chlorine experiment. With a threshold similar to the chlorine experiment, the iodine experiment is also mostly sensitive to ${ }^{8} \mathrm{~B}$ neutrinos, plus some low energy ${ }^{7} \mathrm{Be}$ neutrinos and $\mathrm{CNO}$ neutrinos. Because of its similarity with the chlorine experiment, the iodine experiment can be used to check the chlorine capture rate and the time variation of the rate, which have drawn controversies so far. The advantage of the iodine experiment is that first, the neutrino capture cross section of the iodine is several times larger than that of the chlorine; and second, with the availability of the technology used in the chlorine experiment, the iodine experiment can be put into operation in a matter of months. It is also argued that backgrounds should be relatively low.

In summary, while the present solar neutrino situation is still ambiguous, the next round of experiments should hopefully resolve the situation.

\section{Acknowledgement}

We thank John Bahcall, Ray Davis, David Dearborn, Toshi Koshiba, Ken Lande, Al Mann, Douglas Morrison and John Wilkerson for valuable discussions. This work is supported by NSF and DoE(nuclear) grant at the University of Chicago, and by the NASA/Fermilab astrophysics program.

\section{References}

[1] John N. Bahcall and Roger K. Ulrich, Rev. Mod. Phys. 60,297 (1988).

[2] John N. Bahcall, Neutrino Astrophysics, Cambridge University Press (1989).

[3] J.K. Rowley, B.T. Cleveland, R. Davis, Jr., in Proc. XIII Int. Conf. Neutrino Phys. and Astrophys. (NEUTRINO'88), eds. J. Schneps et al. (World Scientific, Singapore, 1989), p. 518; K. Lande, 25th Intl. Conf. on High Energy Physics, eds. K.K. Phua and Y. Yamaguchi, Vol. 1, 667 (1990).

[4] K.S. Hirata et al., Phys. Rev. Lett. 65, 1297 (1990); Y. Suzuki, in Frontiers of Neutrino Astrophysics, Proc. Intl. Symp. Neutrino Astrophys., Takayama, Japan, 1992, eds. Y. Suzuki and K. Nakamura, Universal Academy Press (1993).

[5] A.I. Abazov, et al., Phys. Rev. Lett. 67, 3332 (1991); V.N. Govrin, talk at the 26th Intl. Conf. on High Energy Physics, Dallas, 1992.

[6] P. Anselmann, et al., Phys. Lett. B 285, 376 (1992).

[7] S. Turck-Chièze, S. Cahn, M. Cassé, and C. Doom, Ap. J., 335, 415 (1988); S. Turck-Chièze and I. Lopes, Ap. J. 408, 347 (1993).

[8] L. Wolfenstein, Phys. Rev. D17, 2369 (1978); S.P. Mikheyev and A. Yu. Smirnov, Sov. J. Nucl. Phys. 42, 913 (1985); for a review, see T.K. Kuo and James Panteleone, Rev. Mod. Phys. 61, 937 (1989).

[9] V. Barger, R.J.N. Phillips and K. Whisnant, Phys. Rev. D43, 1110 (1991); P.I. Krastev and S.T. Petcov, Phys Lett., B245, 85 (1992).

[10] S.A. Bludman, D.C. Kennedy and P.G. Langacker, Nucl. Phys. B374, 373 (1992).

[11] X. Shi, D.N. Schramm and J.N. Bahcall, Phys. Rev. Lett., 69, 717 (1992).

[12] L.B. Okun, M.B. Voloshin, and M.I. Vysotsky, Sov. J. Nucl. Phys., 44, 440 (1986).

[13] C.-S. Lim and W.J. Marciano, Phys. Rev. D37, 1368 (1988).

[14] X. Shi, D.N. Schramm, R. Rosner and D.S. Dearborn, Comments on Nuclear and Particle Physics, Vol. 21, No. 3, 151 (1993).

[15] A. Acker, S. Pakvasa and J. Pantaleone, Phys. Rev. D43, 1754 (1991).

[16] J.N. Bahcall and M.H. Pinsonneault, Rev. Mod. Phys. Vol. 64, No. 4, 885 (1992).

[17] R. Sienkiewicz, J.N. Bahcall, and B. Paczyński, Ap. J., 348,641 (1990).

[18] B.W. Filippone and D.N. Schramm, Ap. J., 253, 393 (1982).

[19] D.R.O. Morrison, Particle World, 3, 30 (1992); D.R.O. Morrison, CERN-PPE/92-109. 
[21] For a review of current and future solar neutrino experiments, see Ch. 10-14 of ref. 2.

[22] J.K. Rowley, B.T. Cleveland and R. Davis, Jr., in Solar Neutrinos and Neutrino Astronomy, eds. M.L. Cherry, K. Lande and W.A. Fowler, AIP conf. Proc. No. 126, AIP, New York, 1984, p. 1.

[23] Calculated from K. Lande's paper of ref. 3.

[24] R. Davis, Jr., in Proc. of 7th Workshop on Grand Unification, ICOBAN'86, 1987, Toyama, Japan, ed. J. Arafune (World Scientific) p. 237.

[25] J.N. Bahcall, G.B. Field and W.H. Press, Ap. J., 320, L69 (1987).

[26] B.W. Filippone, P. Vogel, Phys. Lett. B, 246, 546 (1990); P. Vogel, Workshop on Neutrino Physics, ed. M. BaldoCeolin, Palazzo Loredan, Venice, 23 (1991).

[27] Appendix of ref. 14.

[28] J.W. Bieber et al., Nature, V348, 407 (1990).

[29] J. Straus, J.B. Blake and D.N. Schramm, Ap. J., 204, 481 (1976).

[30] J.N. Bahcall et al., Ap. J. Lett., 292, L79 (1985).

[31] T.A. Tombrello, Nucl. Phys., 71, 459 (1965).

[32] F.C. Barker, Phys. Rev. C28, 1407 (1983).

[33] C.W. Johnson et al., Ap. J., 392, 31420 (1992).

[34] R.W: Kavanagh et al., Bull. Am. Phys. Soc., 14, 1209 (1969).

[35] B.W. Filippone et al., Phys. Rev. C28, 2222 (1983)

[36] Calculated from ref. 1, ref. 7 and ref. 16.

[37] E. Anders and N. Grevesse, Geochim. Cosmochim. Acta, 53, 197 (1989).

[38] D.S. Dearborn, priv. comm.

[39] J.N. Bahcall and H.A. Bethe, Phys. Rev. Lett. 65, 2233 (1990).

[40] S.A. Bludman et al., Phys. Rev. D47, 47 (1993).

[41] T.J. Loredo, Maximum Entropy and Bayesian Methods, ed. P. Fougère, Kluwer Academic Publishers, Dordrecht, 1990, p. 81.

[42] A.J. Baltz and J. Weneser, Phys. Rev. D37, 3364 (1988).

[43] K.S. Hirata et al., Phys. Rev. Lett. 66, 9 (1991).

[44] K. Enquist et al., Nucl. Phys. B349, 754 (1991); X. Shi, D.N. Schramm and B.D. Fields, Phys. Rev. D48, 2563 (1993).

[45] R.S. Raghavan, 1990, Proc. 25th Int. Conf. High Energy Physics, Singapore, eds. K.K. Phua and Y. Yamaguchi (World Scientific, Singapore), Vol. 1, p. 482.

[46] K. Lande, priv. comm.

\section{Addresses:}

X. Shi

The University of Chicago,

Chicago, IL 60637-1433

D.N. Schramm

The University of Chicago,

Chicago, IL 60637-1433

NASA/Fermilab Astrophysics Center, Fermi National Accelerator Laboratory, Box 500, Batavia, IL 60510-500 